\title{
Hepatomegaly in Type 1 Diabetes Mellitus: When to Suspect of Glycogenic Hepatopathy?
}

\author{
Joana Jardim $^{\mathrm{a}, \mathrm{c}}$, Eunice Trindade ${ }^{\mathrm{a}}$, Fatima Carneiro ${ }^{\mathrm{b}}$, Jorge Amil Dias ${ }^{\mathrm{a}}$
}

\begin{abstract}
Uncontrolled type 1 diabetes mellitus is associated with recognized short- and long-term complications. The authors describe a case of a female adolescent, with history of poorly controlled type 1 diabetes mellitus, who presented with tender hepatomegaly and elevated liver enzymes. Her growth and pubertal development were appropriated for age. After excluding infectious and autoimmune causes of liver disease, a liver biopsy was performed and histology revealed glycogenosis. Glycogenic hepatopathy is characterized by abnormal glycogen accumulation in hepatocytes. It is a reversible condition, with good glycemic control, and is not known to progress to fibrosis. This entity should be distinct from other causes of hepatomegaly and elevated liver enzymes, in diabetic patients, such as nonalcoholic fatty liver disease. Although glycogenic hepatopathy is a major cause of hepatomegaly in type 1 diabetes mellitus, is rare and likely under-recognized, the diagnosis has implications in the management and outcome.
\end{abstract}

Keywords: Glycogenic hepatopathy; Diabetes mellitus; Hepatomegaly

\section{Introduction}

In the clinical setting of diabetes, hepatomegaly can be caused by hepatic glycogenosis or nonalcoholic fatty liver disease (NAFLD) [1]. Glycogenic hepatopathy (GH) is a rare and under-recognized condition associated with poorly controlled type 1 diabetes mellitus (T1DM) in children, ado-

\footnotetext{
Manuscript accepted for publication October 2, 2013

${ }^{\mathrm{a} D e p a r t m e n t ~ o f ~ P e d i a t r i c ~ G a s t r o e n t e r o l o g y, ~ C e n t r o ~ H o s p i t a l a r ~ S a o ~ J o a o, ~}$ Oporto, Portugal

${ }^{\mathrm{b}}$ Department of Pathology, Centro Hospitalar Sao Joao, Oporto, Portugal

${ }^{\mathrm{c} C}$ Corresponding author: Joana Isabel de Noronha Lima Jardim da Pena, Alameda Prof. Hermani Monteiro, 4200-319 Oporto, Portugal.

Email: joanajardim@sapo.pt
}

doi: http://dx.doi.org/10.4021/jmc1516w lescents and young adults, while NAFLD is more frequent in older and obese patients with type 2 diabetes $[1,2]$. GH consists in glycogen accumulation in the hepatocytes, resulting in hepatomegaly and elevated liver enzymes [2-6]. The clinical course is benign and $\mathrm{GH}$ is reversible if glycemia is controlled [1-9].

\section{Case Report}

A 14-year-old female was referred to the pediatric gastroenterology outpatient clinic due to chronic abdominal pain and intermittent hepatomegaly. She had T1DM diagnosed since 11 years of age, under insulin therapy, but with poor glycemic control, hemoglobin $A_{1 \mathrm{C}} 13 \%$.

There was no history of liver disease, blood transfusion, exposure to toxins, medication and radiation or cholelithiasis.

She was frequently nauseated and had abdominal pain referred to the right upper quadrant. The patient denied history of jaundice, pruritis, weight loss, hematemesis or rectal bleeding. Past medical history did not reveal additional relevant information.

On physical examination she had tender hepatomegaly, extending $2 \mathrm{~cm}$ below the right costal margin. Her weight was on the 25 th-50th centile and 10th-25th for height. Pu-

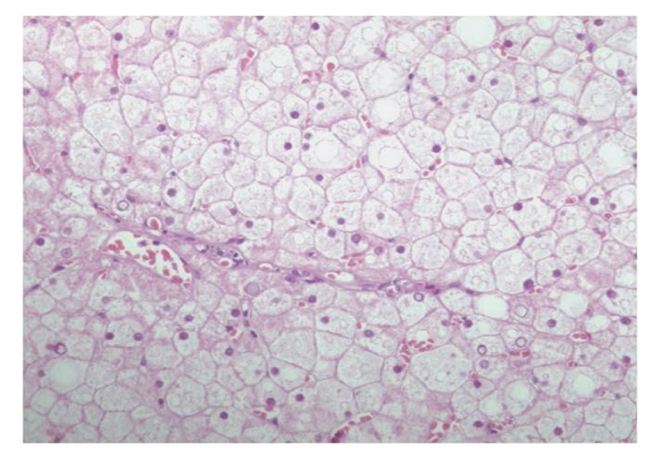

Figure 1. Hepatocytes are diffusely swollen, with clear cytoplasm and sinusoids are collapsed, both features conferring a vegetal-like appearance to liver parenchyma (HE, original magnification $\times 100$ ). 


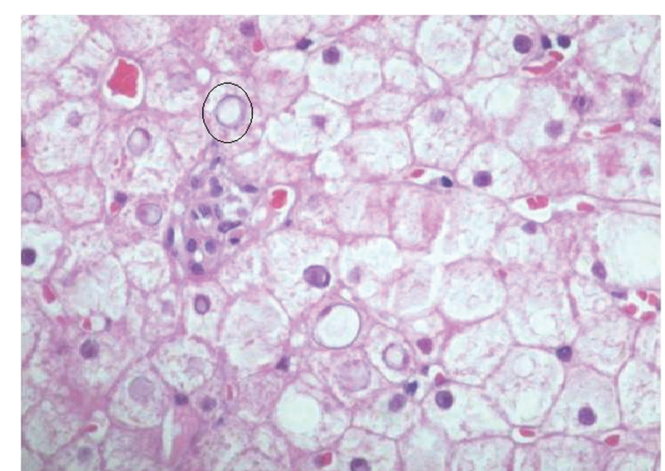

Figure 2. Lipid droplets are identified in the cytoplasm of some hepatocytes and some nuclei display an empty appearance (circle), due to the accumulation of glycogen (original magnification $\times 200$ ).

bertal development was normal. Physical examination was otherwise unremarkable.

Abdominal ultrasound showed liver enlargement (23.7 $\mathrm{cm}$ span) with heterogeneous texture and without evidence of splenomegaly or ascites.

She had elevated aspartate aminotransferase $226 \mathrm{U} / \mathrm{L}$, alanine aminotransferase $109 \mathrm{U} / \mathrm{L}$, gamma-glutamyl transpeptidase $117 \mathrm{U} / \mathrm{L}$ and abnormal lipid profile, total cholesterol $2.52 \mathrm{~g} / \mathrm{L}$ and low density lipoprotein $1.74 \mathrm{~g} / \mathrm{L}$.

Complete blood cell count, bilirubin, liver function panel, serum ferritin level and thyroid function were normal. Serology for HIV, hepatitis A, B and C, cytomegalovirus and Epstein-Barr virus were negative.

Other causes of immune and metabolic hepatic diseases where excluded: serum ceruloplasmin, copper, alpha 1-antitrypsin and immunoglobulin A levels were normal; antitransglutaminase IgA, antinuclear smooth muscle antibody and anti-liver kidney microsomal antibodies were negative.

During the following 2 years, she maintained poorly controlled diabetes, as she refused adhering to the therapy, despite multidisciplinary interventions. At this point, the consistently elevated liver enzymes with severe flares (5 to 6 times above normal) led to liver biopsy that revealed normal trabecular architecture, without evidence of fibrosis, necrosis or inflammation. The hepatocytes were swollen, with abundant clear cytoplasm due to accumulation of glycogen, the cell membranes were thickened and the sinusoids were collapsed, conferring a vegetal-like appearance to liver parenchyma (Fig. 1, 2). A diagnosis of hepatic glycogenosis secondary to poorly controlled diabetic disease was suggested. After biopsy, clinical improvement was only seen when glycemic control was achieved, later on.

\section{Discussion}

$\mathrm{GH}$ results from the accumulation of excessive glycogen in the hepatocytes [1-9]. Typically, it is associated with uncontrolled T1DM, although it has been recently reported in type $2[6,8]$. GH presents with abdominal pain, nauseas, vomiting, hepatomegaly and elevated liver enzymes [2-6]. Glycogen loading of the liver was first described as a component of Mauriac syndrome: growth retardation, hepatomegaly, cushingoid features and delayed puberty $[1,2,5,8,9]$. However, it is now known that GH can develop without the full spectrum of the features of Mauriac syndrome [1, 5, 8, 9].

In the clinical setting of diabetes, it is important to distinguish GH from NAFLD, in patients with abnormal liver tests. Persistent mild alterations in liver enzymes are more in favor of NAFLD, whereas aminotransferase flares are more suggestive of GH [8]. Contrary to NAFLD, GH is not known to progress to fibrosis and the hepatic changes are reversible with adequate glycemic control $[2,3,6,8]$.

The wide fluctuation in glucose and insulin levels is an essential element in the pathophysiology of GH. High levels of glucose cause an insulin independent inflow of glucose into the hepatocytes where it is rapidly phosphorilated and trap in the cell. Subsequent treatment of high glucose levels with insulin causes the trapped glucose to polymerize to glycogen. Glycogen production persists for some time after insulin levels are declined [8]. In NAFLD lipid accumulation results of chronic hyperinsulinemia and hepatocellular insulin resistance [10].

On liver biopsy, GH displays a characteristic vegetallike appearance of the hepatocytes, with no evidence of fibrosis, necrosis or inflammation $[3-5,7]$. The need for liver biopsy, in this context, is a debated issue. Some authors favor the need of liver biopsy to make the distinction between GH and NAFLD, to obtain information for the prediction of prognosis, as pathobiology and therapy are different $[1,2,6$, 8]. On the other hand, some recent publications suggest that, although NAFLD cannot be excluded by history, physical examination or ultrasound, invasive evaluations should be postponed until the response of metabolic control is accomplish $[1,2]$. In our case, liver biopsy was performed in order to differentiate the pathological findings, as glycemic control was very difficult to achieve, while the patient persistently maintained symptomatic hepatomegaly and abnormal liver biochemistry.

It is important that pediatricians be aware of $\mathrm{GH}$ and the association with poorly controlled T1DM, as insulin-reversible hepatic glycogen accumulation is the most common cause of hepatomegaly and abnormal liver biochemistry, in children and young adults with insulin-dependent diabetes mellitus.

\section{Grant Support}

The authors declare that there was no financial support, including governmental assistance and pharmaceutical com- 
pany support, involved in this research.

\section{Conflict of Interest}

The authors declare that there is no conflict of interest.

\section{References}

1. Abaci A, Bekem O, Unuvar T, Ozer E, Bober E, Arslan N, Ozturk Y, et al. Hepatic glycogenosis: a rare cause of hepatomegaly in Type 1 diabetes mellitus. J Diabetes Complications. 2008;22(5):325-328.

2. Munns CF, McCrossin RB, Thomsett MJ, Batch J. Hepatic glycogenosis: reversible hepatomegaly in type 1 diabetes. J Paediatr Child Health. 2000;36(5):449-452.

3. Saxena P, Turner I, McIndoe R. Education and Imaging. Hepatobiliary and pancreatic: Glycogenic hepatopathy: a reversible condition. J Gastroenterol Hepatol. 2010;25(3):646.

4. Bassett JT, Veerappan GR, Lee DH. Glycogenic hepatopathy: a rare cause of increased aminotransferase levels in a diabetic patient. Clin Gastroenterol Hepatol. 2008;6(11):A26.

5. Sweetser S, Kraichely RE. The bright liver of glycogenic hepatopathy. Hepatology. 2010;51(2):711-712.

6. Saadi T. Glycogenic hepatopathy: a rare disease that can appear and resolve rapidly in parallel with glycemic control. Isr Med Assoc J. 2012;14(4):269-270.

7. Hudacko RM, Manoukian AV, Schneider SH, Fyfe B. Clinical resolution of glycogenic hepatopathy following improved glycemic control. J Diabetes Complications. 2008;22(5):329-330.

8. van den Brand M, Elving LD, Drenth JP, van Krieken JH. Glycogenic hepatopathy: a rare cause of elevated serum transaminases in diabetes mellitus. Neth J Med. 2009;67(11):394-396.

9. Aljabri KS, Bokhari SA, Fageeh SM, Alharbi AM, Abaza MA. Glycogen hepatopathy in a 13-year-old male with type 1 diabetes. Ann Saudi Med. 2011;31(4):424427.

10. Vo HTD, Klein GW, Loizides A, Zhou P, Liu Q, Pan DH. Glycogen hepatopathy in children with poorly controlled type 1 diabetes. International Journal of Case Reports and Images. 2011;2(9):1-4. 